\title{
Urgences
}

\section{Judith Thurman, Karen Blixen, Paris, Seghers, 1986, 504 p.}

\section{Guy Rancourt}

Numéro 15, octobre 1986

Épigraphiques

URI : https://id.erudit.org/iderudit/025363ar

DOI : https://doi.org/10.7202/025363ar

Aller au sommaire du numéro

Éditeur(s)

Urgences

ISSN

0226-9554 (imprimé)

1927-3924 (numérique)

Découvrir la revue

Citer ce compte rendu

Rancourt, G. (1986). Compte rendu de [Judith Thurman, Karen Blixen, Paris,

Seghers, 1986, 504 p.] Urgences, (15), 113-115. https://doi.org/10.7202/025363ar

Ce document est protégé par la loi sur le droit d'auteur. L’utilisation des services d’Érudit (y compris la reproduction) est assujettie à sa politique d'utilisation que vous pouvez consulter en ligne.

https://apropos.erudit.org/fr/usagers/politique-dutilisation/
Cet article est diffusé et préservé par Érudit.

Érudit est un consortium interuniversitaire sans but lucratif composé de l’Université de Montréal, l’Université Laval et l’Université du Québec à Montréal. Il a pour mission la promotion et la valorisation de la recherche. https://www.erudit.org/fr/ 
entre le début des années 1950 et le début des années 1970. Livre fort soucieux de son écriture, chaleureux, passionnant, dont les personnages, avec ou sans leur troupe ambulante, leur club, les Dodgers, sont des personnes qui, chacune à leur façon, disent rêves et déceptions, ambitions et meurtrissures, leur venue au sport et au monde - et la difficulté du retour, du retour sur soi au passé comme au présent. Et si les (ex-)athlètes ont bien sûr la part belle, Carl Erskine, Gil Hodges, Clem Labine, Joe Black. Carl Furillo, Roy Campanella, Duke Snider, Pee Wee Reese, Jackie Robinson (surtout peut-être) et d'autres encore, Roger Kahn sait aussi évoquer Robert Frost, Shakespeare, et James Joyce discutant de Hamlet dans Ulysse. Vous aimez la littérature et le baseball? Lisez The Boys of Summer (qui connut, il faut le dire, un énorme succès lors de sa parution. De même que cet autre livre plus récent, $B$ ums de Peter Golenbock publié en 1984. Les Dodgers fascinent, semble-t-il; à cause du destin particulier, voire tragique même, de certains des bums?).

Deux remarques en terminant. Duke Snider? Oui, oui, il s'agit bien de celui qui commente les matches des Expos au réseau anglais de Radio-Canada. Le titre du livre? Ce numéro d'Urgences oblige - en épigraphe au livre de Kahn, les lignes suivantes de Dylan Thomas:

I see the boys of summer in their ruin Lay the gold tithings barren, Setting no store by harvest, freeze the soils.

Renald Bérubé

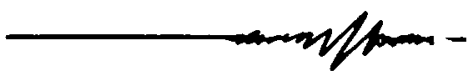

\section{Judith Thurman: Karen Blixen, Paris, Seghers (Biographie), 1986 , 504 p.}

Cette excellente biographie parut d'abord en anglais sous le titre Isak Dinesen. The Life of a Storyteller en 1982. Karen Blixen? Isak Dinesen? Que l'on ne se surprenne pas, il s'agit bel et bien de la même auteure, et on pourrait continuer la liste des pseudonymes qu'elle affectionnait tant. Pourquoi pas celui d'Osceola, nom sous lequel elle fit paraître ses tout premiers contes (1907)? Ou encore celui de Pierre Andrézel, pseudonyme français qu'elle emprunta lors de la publication de son roman Les voles de la vengeance (1944)? Qui est donc cette femme aux multiples appellations à qui le plus récent film de Sydney Pollack, Out of Africa, semble assurer un regain de popularité? C'est précisément à cette découverte que Judith Thurman nous convie dans son magnifique ouvrage sur celle qui fut pour la littérature danoise l'équivalent de ce qu'on été Sigrid Undset pour la norvégienne et Selma Lagerlöf pour la suédoise.

$$
* *
$$

Karen Blixen (pour les lecteurs français et danois) ou Isak Dinesen (pour les lecteurs anglophones) est née le 17 avril 1885 à Rungstedlund, près de Copenhague. Elle fut baptisée sous le nom de Karen Christentze Dinesen mais très tôt dans sa famille, on l'appela "Tanne", diminutif qu'elle abhorrait et qui venait de sa prononciation enfantine de Karen. Plus tard, elle deviendra la baronne $\mathrm{Ka}$ ren Blixen-Finecke lorsqu'elle se mariera, en 1914, avec un cousin suédois, Bror Blixen-Finecke (ce dernier était un ami d'Ernest Hemingway qui le dépeignit dans un de ses textes, "The Short Happy Life of Francis Macomber" sous les traits d'un chasseur blanc, Robert Wilson). Mariage improvisé et malheureux qui se termine par une rupture ( $\mathrm{di}$ vorce) en 1922. Puis, en 1934, elle signera sa première oeuvre importante Sept contes gothiques sous le nom d'lsak Dinesen. Ses nombreux admira 
teurs lui donnèrent une multtude de surnoms: "Lord Byron", "Khamar", "Pellegrina", "Schéhérazade", etc. Enfin, après une carrière littéraire bien remplie, elle va s'éteindre à l'áge de 77 ans, le 7 septembre 1962 dans sa patrie natale, des sultes d'une longue maladie (syphilis). Sur sa pierre tombale figure le nom de Karen Blixen!

ॠい*

Comment Judith Thurman nous présente-t-elle la vie de Karen Blixen? Sa biographie comporte 52 chapitres découpés en quatre grandes sections. Le premier livre (14 chapitres) intitulé "Tanne" couvre la période danoise de 1885 à 1914 et expose un tableau précis de son milieu famillal et social, de son enfance et de son adolescence, ainsi que de ses années de formation. Le second livre (18 chapitres), "Tania" (surnom que lui donnerent les Africains), fouille en détail son long séjour en Afrique (Kenya) qui va de 1914 a 1931. Cette expérience africaine si riche en événements de toutes sortes restera un des éléments marquants à la fois de sa personnalité et de son oeuvre littéraire. Le troisième livre (14 chapitres), "Isak Dinesen", raconte les principales étapes de sa carrière d'écrivain de 1931 à 1955. Enfin le demier livre $(6$ chapitres), "Pellegrina", beaucoup plus court, présente les six dernières années (1956-1962) de celle qui était devenue une légende vivante (de son "mythe" comme elle disait elle-méme) dans le paysage littéraire européen. Un chapitre est à retenir spécialement dans cette dernière section: "Isak Dinesen en Amérique" où sont décrites ses rencontres avec une foule d'écrivains américains (Truman Capote, Cecil Beaton, Steinbeck, le poète Arthur Gregor, Pearl Buck...) et surtout sa fameuse rencontre avec Carson McCullers, Arthur Miller et Marilyn Monroe en février 1959. Et comme Karen Blixen tenait à rencontrer personnellement cette dernière!
Ce qu'il importe de ne pas perdre de vue dans cette biographie, ce sont les deux données majeures autour desquelles peut s'articuler toute l'oeuvre litteraire de Karen Blixen. 1) Il s'agit d'abord de son expérience africaine (1914-1931): cela donne Out of Afrt. ca (1937); Ombres sur la prairie (1960), récit composé de 4 nouvelles qui est, somme toute, l' épilogue de Out of Africa; Lettres d'Afrique (1978) maintenant traduites en français chez Gallimard (1985), oeuvre posthume qui rassemble la plupart des lettres que Karen Blixen a écrites lors de son séjour au Kenya. Ce texte est important pour qui veut connaitre la face cachée, le négatif en quelque sorte de son expérience africaine trop idéalisée et déréalisée par son récit Out of Africa, et comblen romantisée dans le film de Pollack! (À ce sujet, il faut lire la biographle de Denys Finch Hatton rédigée par Errol Trzebinski, SIlence Will Speak (1977), pour constater que la relation amoureuse Karen/Denys fut loin d'etre aussi idyllique...). 2) Ensuite, vient la seconde donnée, l'art d'inventer des histoires (la Karen Blixen conteuse): cela donne Sept contes gothiques (1934), Contes d'hiver (1942) qui était son texte préféré, Nouveaux contes d'ht. ver (1957) et Le diner de Babette (1958), dans lequel on trouve "Histoire immortelle" qui, de ses dernieres oeuvres, était sa favorite.

******

La biographie rédigée par Judith Thurman est stimulante à lire à bien des égards. Primo, elle nous permet une plongée dans l'univers littéraire scandinave (danois en particulier). On a le goût de relire les philosophes Kierkegaard et Brandés qui ont façonné en quelque sorte la mentalité de leurs concitoyens. Secondo, on a le gout de relire Andersen, car il y a du Andersen chez la Karen Blixen conteuse, pas seulement de la Schéhérazade! Tertio, cela 
nous incite à vouloir lire les poètes danois. Heureusement qu'il existe en français, et cela depuis 1975, une excellente Anthologie de la poésie danolse contemporaine publiée chez Gallimard sous les soins de Jorgen Gustava Brandt, Uffe Harder et Klaus Rifbjerg. Vous pourrez y lire des poètes teis que Sophus Claussen (1865-1931), poète préféré de Karen Blixen, ou encore Thorkild Bjomvig (1918- ) son ami de coeur et protégé. Enfin, en plus de mieux connaitre cette auteure, vous allez avoir le gout de lire toute son oeuvre...

Guy Rancourt

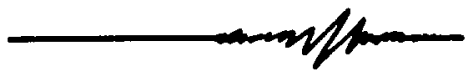

\section{Dominique Noguez:} Les trois Rimbaud, Paris, Minuit, 1986, $62 \mathrm{p}$.

Qui ne connaît pas ce cher Arthur, qui écrivit (toute! toute?) son oeuvre de 1869 à 1875-1876, de 15 ans à 21-22 ans. Ou son "cousin" d'outreAtlantique Emile, qui écrivit (id.! id.?) son oeuvre de 1896 à 1899 , de 16 à 19 ans.

Qui ne connaît pas "La chasse spirituelle", cette fausse prose à lui attribuée, une affaire qui défraya la chronique parisienne en 1949. Voir, là-dessus, le pamphlet d'André Breton: "Flagrant délit" (dans La clé des champs, coll. "10/18", 750, [1953], p. 209-273).

Qui ne connaît pas, enfin, "la plaisante fiction d'Alain Borer qui, dans son Rimbaud en Abyssinie [en note: Paru en 1984 aux Éditions du Seuil, dans la collection, bien nommée, Fiction \& Cie...], prête à l'écrivain le destin de son personnage des Nuits d'Afrique, le fait donc mourir vers 1891 des suites d'une amputation de la jambe, et s'amuse ensuite à imaginer l'idée que nous nous ferions aujourd'hui de lui si, à Dieu ne plat, tel avec été le cas (petit jeu littéraire qui ne manque pas d'intérêt et qui vaudrait d'être pratiqué sur d'autres: comment verrions-nous Gide s'il était mort après Les Nourritures terrestres, Aragon après le Traité du style ou Joyce après The Dubltners?...)" (p. 27).

Il aura donc fallu attendre Dominique Noguez pour indiquer la "vole comparative" (p. 62) nécessaire à la relecture de l'oeuvre une, certes, mais selon les deux majeures coupures qu'il est désormais convenu d'envisager, "pour ce qui est de la vie" (p. 13):

- le premier Rimbaud, "trop souvent oublié" (p. 30): de sa naissance (1854) a 1875; celui des premières fugues et des oeuvres d'adolescence: Poésies, Vers nouveaux et chansons (fin 1871-1872), Une saison en enfer (1873), Illuminations;

- le deuxième Rimbaud: de 1875 1876 à 1891: celui du "silence" littéraire, des longs voyages en Europe, un peu en Orient et, surtout, des longues explorations en Afrique, jusqu' au retour incognito à Paris:

- le troisième Rimbaud: de 18911893 à sa mort (1937): celui des "rechutes" - les guillemets sont de D.N. (p. 24) - presque officielles (en Chine, aux États-Unis) et des oeuvres importantes et bien connues de la maturité et de la vieillesse: Les Nuits d'Afrique (1893), Système de la vie modeme (1899-1911, inachevé), $\boldsymbol{L}$ (1910), $\boldsymbol{L} \cdot \dot{E}$. vangile noir (1928) et Regards sans yeux (1934).

L'intérêt de cette brève étude. illustrée de quelques documents, est bien de replacer les grandes oeuvres bien connues, trop (mal) connues - n'ontelles pas mené leur auteur à l'Académie française (1930), où il est reçu par Valéry - dans la contradictoire continuité d'Une saison en enfer, surtout, "texte décidément capital" (p. 58):

En somme, le vrai lieu de Rimbaud est la racine brúlante de la contradic- 